\title{
Hybridization Concepts of Artificial Human Optimization Field Algorithms Incorporated into Particle Swarm Optimization
}

\author{
Hassan M. H. Mustafa \\ Faculty of Specified Education, Dept. of \\ Educational Technology, Banha University, Egypt
}

\author{
Satish Gajawada \\ Alumnus, Indian Institute of Technology Roorkee \\ Founder, Artificial Human Optimization Field
}

\begin{abstract}
This piece of research presents the Particle Swarm Optimization (PSO) as a biologically inspired computational paradigm searches for problem optimization technique. Specifically (PSO) consists of a swarm of particles, where particle represent a potential solution. More precisely it is a population-based, stochastic algorithm modeled on the social behaviors observed in flocking birds. Over the past quarter century, Global Optimization techniques like Genetic and PSO algorithms has attracted many researchers attention at engineering and industry. In December 2016, a new field titled "Artificial Human Optimization" was introduced in literature. Referring to that innovative field it is clear that the agents in Artificial Human Optimization are Artificial Humans. Recently, a new algorithm titled Multiple Strategy Human Optimization (MSHO) is designed based on Artificial Humans. This paper adopted an interesting novel experimental idea which incorporated Hybridization of perspective concepts of Artificial Human Optimization into some experimental illustrations of PSO algorithms. Additionally, Human Inspired Differential Evolution (HIDE) is recently proposed method which is based on Differential Evolution and MSHO. For particular parameters settings HIDE performed approximately as good as Differential Evolution. In the experiment in this paper, a new algorithm titled "Hassan Satish Particle Swarm Optimization (HSPSO)" is proposed. HSPSO is tested by applying it on a complex benchmark function. Interesting Hybridization results have been obtained. Results obtained by HSPSO are compared with Particle Swarm Optimization.
\end{abstract}

\section{General Terms}

Evolutionary Computing, Global Optimization Techniques, Nature Inspired Computation, Bio-Inspired Computing, Machine Learning, Artificial Intelligence.

\section{Keywords}

Hybridization, Particle Swarm Optimization, Artificial Human Optimization, Genetic Algorithms, Differential Evolution, Hybrid Algorithms.

\section{INTRODUCTION}

This research work is essentially originated from the optimization techniques adopted for solving optimally complex problems specified with necessary features and functions. Furthermore, these problems employed with an adaptive, adjustable infrastructure, in addition to their spontaneous and ubiquitous problematic features. In this context, any optimization technique can be defined as an art of selecting the best alternative among a given set of options. Furthermore, it is motivated originally by Mathematics and Computer Science domains aiming to find the best element among set of available elements.
More specifically, Particle Swarm Optimization (PSO) is a biologically inspired computational search and optimization method developed in 1995 by Eberhart and Kennedy [1], based on the social behaviors of birds flocking or fish schooling.

Additionally, research work at the field of Optimization problems forms an integral part of our day-to-day life. In the most basic sense, it can be defined as an art of selecting the best alternative among a given set of options. Moreover, Optimization problems arise in various disciplines such as engineering designs, agricultural sciences, manufacturing systems, economics, physical sciences, pattern recognition etc. In fact optimization techniques are being extensively used in various spheres of human activities, where decisions have to be taken in some complex situation that can be represented by a mathematical model. Optimization can thus be viewed as one of the major quantitative tools in network of decision making, in which decisions have to be taken to optimize one or more objectives in some prescribed set of circumstances. In view of the practical utility of optimization problems there is a need for efficient and robust computational algorithms, which can numerically solve on computers the mathematical models of medium as well as large size optimization problem arising in different fields. In the past few decades several global optimization algorithms have been developed that are based on the nature inspired analogy. These are mostly population based metaheuristics also called general purpose algorithms because of their applicability to a wide range of problems. The following four distinct characteristic aspects concerned with PSO searching for "best available" solution for either unimodal or multimodal optimization problem:

- Particle Swarm Optimization (PSO) is a simple but powerful search technique.

- PSO applies the concept of social interaction to problem solving.

- In PSO, a swarm of $\mathrm{n}$ individuals communicate either directly or indirectly with one another search directions (gradients).

- It has been applied successfully to a wide variety of search and optimization problems.

The rest of this paper is organized as follows. At the next second section a brief literature review is presented. It included variety of optimization challenging problems, based mainly on hybridization of PSO. Detailed procedure of PSO is revised at the third section, along with some simple illustrations of the algorithmic steps. At the fourth section, a suggested novel algorithm titled "Hassan Satish Particle Swarm Optimization (HSPSO)" is introduced. A comparison between PSO and HSPSO considering ACKLEY function is given at the fifth section. Finally, at the sixth section some conclusive remarks have been given at the end of this manuscript. 


\section{A LITERATURE REVIEW [6][7]}

In reality, the researchers at the field of optimization problems techniques who adopted PSO approach, have considered Hybridizing/combining techniques of swarm Intelligence optimization, PSO as well into some developed genetic and differential evolution techniques. In other words, Hybridization is a method of combining two (or more) techniques in a judicious manner such that the resulting algorithm contains the positive features of both (or all) the algorithms. Depending on the algorithms used, there can be three classifications as (i) Hybridization of PSO and genetic algorithms (ii) Hybridization of PSO with differential evolution and (iii) Hybridization of PSO with other techniques. Two hybrid PSO algorithms; hybrid differential evolution particle swarm optimization (DE-PSO), and hybrid genetic algorithm particle swarm optimization (GA-PSO). More precisely, such optimization techniques are being extensively used in various spheres of human activities, where decisions have to be taken in some complex situation that can be represented by a mathematical model. Optimization can thus be viewed as one of the major quantitative tools in network of decision making, in which decisions have to be taken to optimize one or more objectives in some prescribed set of circumstances. In view of the practical utility of optimization problems there is a need for efficient and robust computational algorithms, which can numerically solve on computers the mathematical models of medium as well as large size optimization problem arising in different fields. In the past few decades several global optimization algorithms have been developed that are based on the nature inspired analogy. These are mostly population based meta-heuristics also called general purpose algorithms because of their applicability to a wide range of problems. Some popular global optimization algorithms include genetic algorithms (GA) [1], particle swarm optimization (PSO) [2], Differential Evolution (DE) [3], evolutionary programming (EP) [4], ant colony optimization ACO) [5] etc. These algorithms have proved their mettle in solving complex and intricate optimization problems arising in various fields of science and technology such as engineering designs, agricultural sciences, manufacturing systems, economics, physical sciences, pattern recognition etc. Furthermore, a chronological order of the development of some of the popular nature inspired metaheuristics is given in Figure 1. These algorithms have proved their mettle in solving complex and intricate optimization problems arising in various fields.

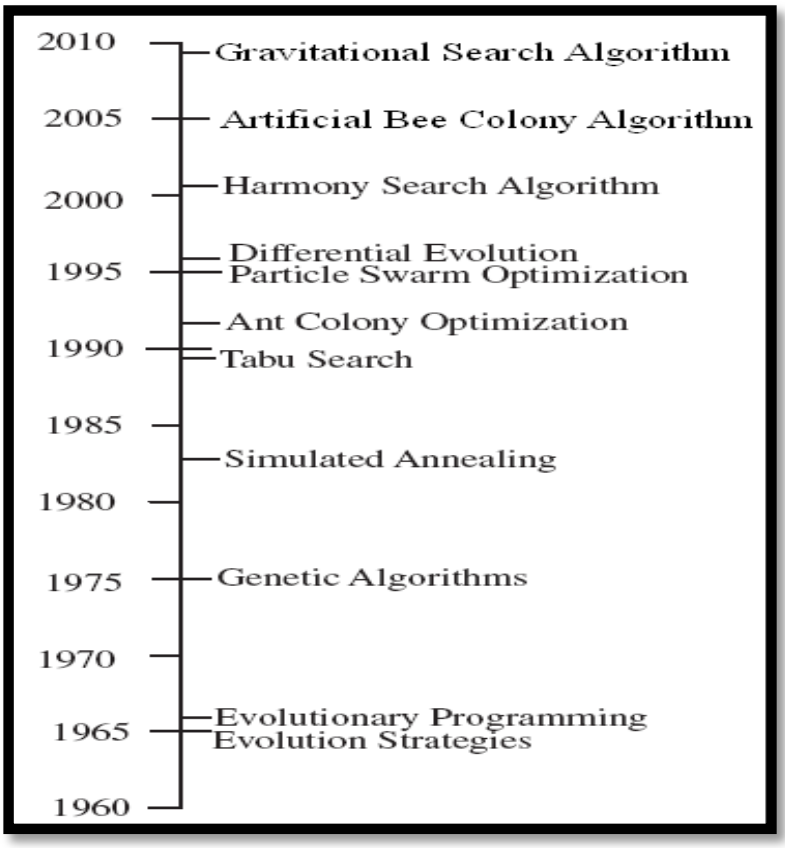

Figure 1: Popular meta-heuristics in chronological order (Adapted from [6])

Referring to [7], Particle swarm optimization (PSO) is a population-based stochastic algorithm modeled on the social behaviors observed in flocking birds. Over the past quarter century, the particle swarm optimization algorithm has attracted many researchers' attention. Through the convergent operation and divergent operation, individuals in PSO group and diverge in the search space/objective space. In this paper, the historical development, the state-of-the-art, and the applications of the PSO algorithms are reviewed. In addition, the characteristics and issues of the PSO algorithm are also discussed from the evolution and learning perspectives. Every individual in the PSO algorithm learns from itself and another particle with good fitness value. A suggested example of an PSO algorithm is presented by the simplified flowchart is shown in below at Figure 2, considering the algorithmic procedure steps given at Fig.2

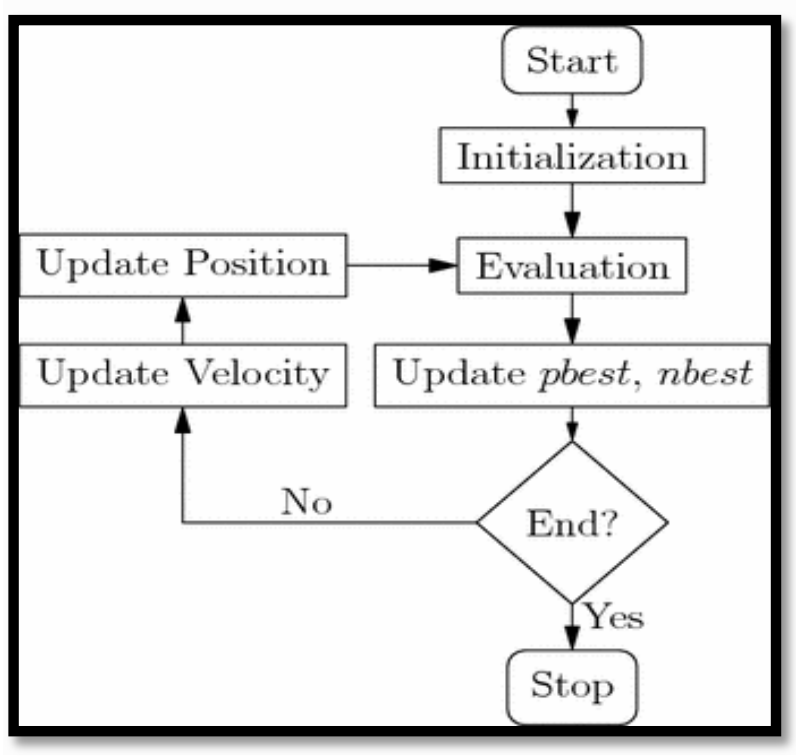

Figure 2: The flowchart of particle swarm optimization algorithm (Adapted from [7]) 
Referring to [7], Pie chart showing the distribution of publication of research articles having hybrid versions of PSO number of hybrid PSO papers published in the year 2001 to 2010 .

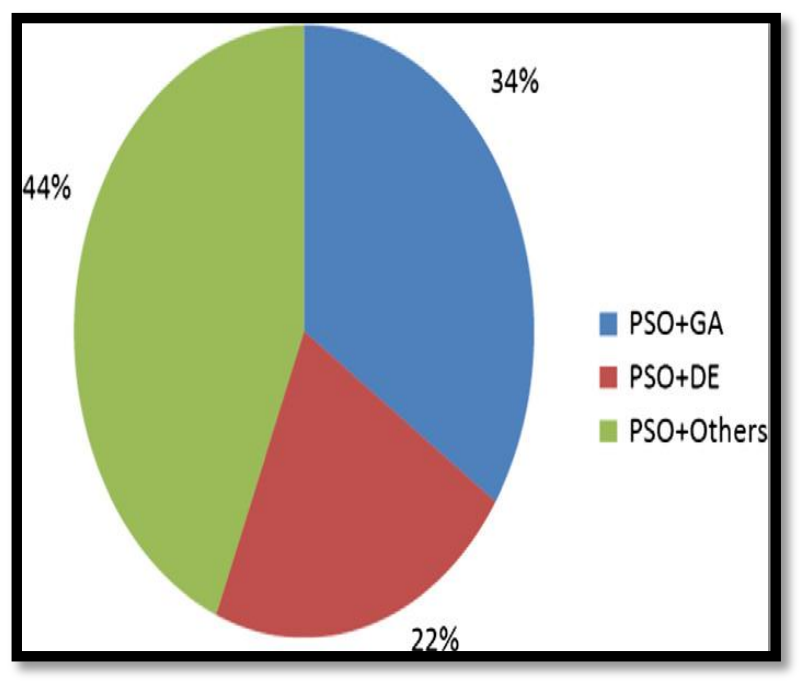

Figure 3: Pie chart showing the publication distribution of the meta-heuristics algorithms

In [8], a very good introduction to Artificial Human Optimization Field was given which is shown below as it is in double quotes: "In [9], Artificial Human Optimization (AHO) was introduced. 10 plus reviews on work in AHO field were shown in [10]. A new algorithm titled "POSTDOC: The Human Optimization" was designed in [11]. In [12], an Introduction to AHO field was given. Ocean of opportunities in Artificial Human Optimization Field was shown in [13]. According to [13], millions of papers are possible in AHO Field. 25 reviews from experts on work under AHO field were given in [14]. 30 plus abstracts of papers in Artificial Human Optimization Field were shown in [15]. The first paper in Artificial Human Optimization Field was proposed in 2006 [16]. In [12], Multiple Strategy Human Optimization (MSHO) was proposed". A Hybrid Optimization method based on AHO concepts and Differential Evolution is proposed recently in [8]. In articles [17] - [21] algorithms were proposed based on AHO concepts and Particle Swarm Optimization. But these algorithms don't use the concepts of Multiple Strategy Human Optimization (MSHO) proposed in [12]. The experiment conducted in this paper is to use the concepts of MSHO into Particle Swarm Optimization.

\section{REVISING OF (PSO) PROCEDURE}

In the present study an attempt is made to review the one main algorithm is a well known meta-heuristic; Particle Swarm Optimization (PSO). PSO, in its present form, has been in existence for roughly a decade, a relatively short time compared with some of the other natural computing paradigms such as artificial neural networks and evolutionary computation. However, in that short period, PSO has gained widespread appeal amongst researchers and has been shown to offer good performance in a variety of application domains, with potential for hybridization and specialization, and demonstration of some interesting emergent behavior. In PSO, first we initialize all particles as shown below. Two variables pbest $_{\mathrm{i}}$ and gbest are maintained. pbest $\mathrm{i}_{\mathrm{i}}$ is the best fitness value achieved by $i^{\text {th }}$ particle so far and gbest is the best fitness value achieved by all particles so far. Lines 4 to 11 in the below text helps in maintaining particle best and global best.
Then the velocity is updated by rule shown in line no. 14 . Line 15 updates position of $i^{\text {th }}$ particle. Line 19 increments the number of iterations and then the control goes back to line 4 . This process of a particle moving towards its local best and also moving towards global best of particles is continued until termination criteria will be reached.

Procedure: Particle Swarm Optimization ( PSO )

1) Initialize all particles

2) iterations $=0$

3) do

4) for each particle i do

6)

If $\left(f\left(x_{i}\right)<f\left(\right.\right.$ pbest $\left.\left._{i}\right)\right)$ then

pbest $_{\mathrm{i}}=\mathrm{x}_{\mathrm{i}}$

end if

$$
\text { if }\left(f\left(\text { pbest }_{i}\right)<f(\text { gbest })\right) \text { then }
$$

$$
\text { gbest }=\text { pbest }_{\mathrm{i}}
$$

end if

11) end for

12) for each particle i do

for each dimension d do

18) end for

19) iterations $=$ iterations +1

20) while ( termination condition is false)

\section{HASSAN SATISH PARTICLE SWARM OPTIMIZATION (HSPSO)}

HSPSO is obtained by incorporation of MSHO concepts into Particle Swarm Optimization. In starting and even generations the Artificial Humans move towards the best fitness value. In odd generations Artificial Humans move away from the worst fitness value. In HSPSO, we maintain local worst of particle and global worst of all particles in addition to local best of particle and global best of all particles. This is shown in lines 4 to 17 . In lines 19 to 24 velocity is calculated by moving towards the local best of particle and global best of all particles. In lines 26 to 31 pseudo code for odd generations is shown in below text. In these odd generations particles move away from local worst of particle and also away from global worst of all particles. In line 33, number of iterations is incremented by one. Then control goes back to line number 4 . This process of moving towards the best in one generation and moving away from the worst in next generation is continued until termination criteria has been reached.

Procedure: Hassan Satish Particle Swarm Optimization (HSPSO)

1) Initialize all particles

2) iterations $=0$

3) do 
4) for each particle i do

18) If $(($ iterations $==0) \|($ iterations $\% 2==0))$ then // for starting and even iterations

for each particle i do

$$
\text { for each dimension } \mathrm{d} \text { do }
$$

$$
\mathrm{v}_{\mathrm{i}, \mathrm{d}}=\mathrm{v}_{\mathrm{i}, \mathrm{d}}+
$$

$\mathrm{C}_{1} * \operatorname{Random}(0,1) *\left(\right.$ pbest $\left._{\mathrm{i}, \mathrm{d}}-\mathrm{x}_{\mathrm{i}, \mathrm{d}}\right)$

$$
+\mathrm{C}_{2} * \operatorname{Random}(0,1) *\left(\text { gbest }_{\mathrm{d}}-\mathrm{x}_{\mathrm{i}, \mathrm{d}}\right)
$$

$$
\begin{aligned}
& \text { end for } \\
& \text { end for }
\end{aligned}
$$

else // for odd iterations

for each particle i do

for each dimension d do

$$
\begin{aligned}
& \mathrm{v}_{\mathrm{i}, \mathrm{d}}=\mathrm{v}_{\mathrm{i}, \mathrm{d}}+ \\
& \mathrm{C}_{1} * \operatorname{Random}(0,1) *\left(\mathrm{x}_{\mathrm{i}, \mathrm{d}}-\right. \\
& \text { pworst } \left._{\mathrm{i}, \mathrm{d}}\right) \\
& +\mathrm{C}_{2} * \operatorname{Random}(0,1) *\left(\mathrm{x}_{\mathrm{i}, \mathrm{d}}-\right. \\
& \text { gworst } \left._{\mathrm{d}}\right)
\end{aligned}
$$$$
\mathrm{x}_{\mathrm{i}, \mathrm{d}}=\mathrm{x}_{\mathrm{i}, \mathrm{d}}+\mathrm{v}_{\mathrm{i}, \mathrm{d}}
$$$$
\text { end for }
$$

end for

$$
\begin{aligned}
& \text { end if } \\
& \text { iterations }=\text { iterations }+1
\end{aligned}
$$

34) while ( termination condition is false)

\section{COMPARISION BETWEEN PSO AND HSPSO FOR ACKLEY FUNCTION}

Figure 4 shows Ackley function 2D. This figure is taken from [22].

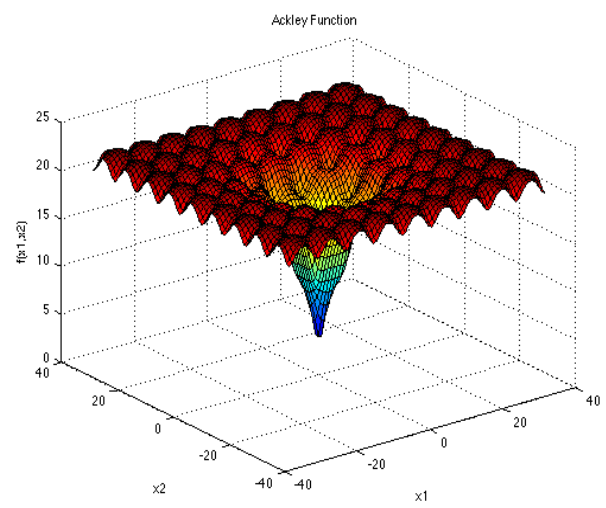

Figure 4. Ackley Function 2D

Figure 5 shows parameter settings. Figure 6 and Figure 7 shows results given by HSPSO and PSO respectively.

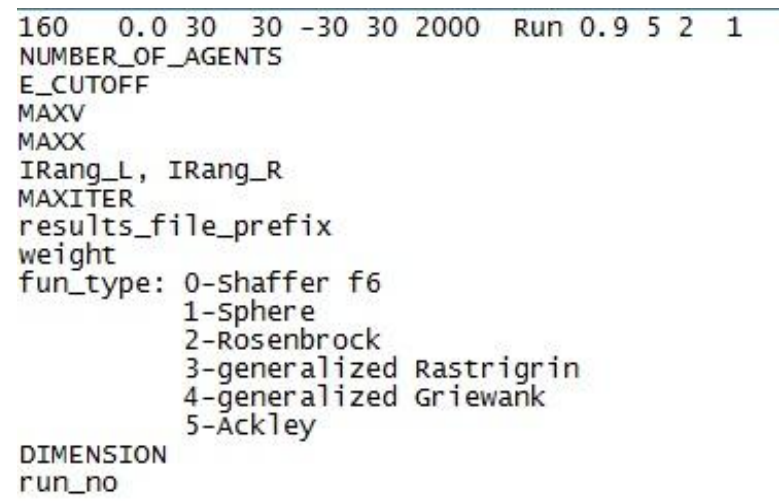

Figure 5. Parameter Settings

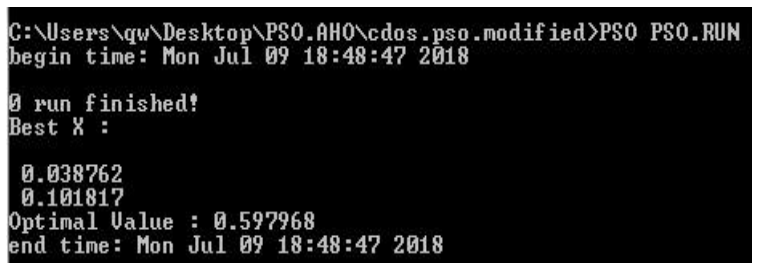

Figure 6. Result given by Hassan Satish PSO ( HSPSO )



Figure 7. Result given by Particle Swarm Optimization ( PSO )

From Figure 6, it can be seen that the optimal solution given by HSPSO is $(0.03,0.10)$ and optimal value is 0.59 . From Figure 7 it can be seen that the PSO algorithm gave optimal solution as $(0,0)$ with optimal value 0 which is exact optimal solution and optimal value. Hence it can be concluded that PSO performed better than proposed HSPSO algorithm for 
this particular parameters setting and particular complex benchmark function.

\section{CONCLUSION}

Multiple Strategy Human Optimization (MSHO) is proposed recently in literature which is based on Artificial Humans [12]. A new algorithm titled "Hassan Satish Particle Swarm Optimization (HSPSO)" is proposed by applying MSHO concepts into Particle Swarm Optimization. The proposed HSPSO algorithm is tested by applying it on a complex benchmark function. PSO performed better than proposed HSPSO algorithm for particular parameter settings and benchmark function. Applying HSPSO on many benchmark functions and comparing results obtained with PSO will be part of future work. General expectation is that optimization methods based on Artificial Humans will perform better than optimization algorithms based on other beings like birds etc. From results obtained in this paper we can conclude that Artificial Human Optimization Algorithms might not always perform better than other optimization algorithms like PSO.

\section{REFERENCES}

[1] J.H. Holland, Adaptation in natural and artificial systems: an introductory analysis with applications to biology, control, and artificial intelligence. University of Michigan Press.

[2] J. Kennedy, R.C. Eberhart, Particle Swarm Optimization, in: Proceedings of the IEEE International Conference on Neural Networks, Piscataway, vol. IV, 1995, pp. 19421948.

[3] K. Price, R. Storn, Differential evolution - a simple and efficient adaptive scheme for global optimization over continuous spaces, Technical Report, International Computer Science Institute, Berkley, 1995.

[4] L.J. Fogel, A.J. Owens, M.J. Walsh, Artificial intelligence through a simulation of evolution, in: $M$. Maxfield, A. Callahan, L.J. Fogel, (eds.), Biophysics and Cybernetic systems, Proceedings of the 2nd Cybernetic Sciences Symposium, 1965, pp. 131-155, (Spartan Books).

[5] M. Dorigo, V. Maniezzo, A. Colorni, Positive feedback as a search strategy, Technical Report 91-016, Dipartimento di Elettronica, Politecnico di Milano, IT, 1991.

[6] Mahdiyeh Eslami, Hussain Shareef, Mohammad khajehzadeh, Azah Mohamed, "A Survey of the State of the Art in Particle Swarm Optimization" Published Research journal of Applied Sciences, Engineering and Technology 4(9): 1181-1197, 2012 ISSN: 2040-7467.

[7] Shi Cheng, Hui Lu, Xiujuan Lei, Yuhui Shi, "Survey and State of the Art quarter century of particle swarm optimization" Available online at https://link.springer.com/article/10.1007/s40747-0180071-2. April 2018.

[8] Satish Gajawada, Hassan M. H. Mustafa , HIDE : Human Inspired Differential Evolution - An Algorithm under Artificial Human Optimization Field, International Journal of Research Publications (Volume: 7, Issue: 1), http://ijrp.org/paper-detail/264
[9] Satish Gajawada; Entrepreneur: Artificial Human Optimization. Transactions on Machine Learning and Artificial Intelligence, Volume 4 No 6 December (2016); pp: 64-70.

[10] Satish Gajawada, "CEO: Different Reviews on $\mathrm{PhD}$ in Artificial Intelligence", Global Journal of Advanced Research, vol. 1, no.2, pp. 155-158, 2014.

[11] Satish Gajawada, "POSTDOC : The Human Optimization", Computer Science \& Information Technology (CS \& IT), CSCP, pp. 183-187, 2013.

[12] Satish Gajawada, "Artificial Human Optimization - An Introduction", Transactions on Machine Learning and Artificial Intelligence Volume 6, No 2, pp: 1-9, April 2018 .

[13] Satish Gajawada, "An Ocean of Opportunities in Artificial Human Optimization Field", Transactions on Machine Learning and Artificial Intelligence, Volume 6, No 3, June 2018.,.

[14] Satish Gajawada, "25 Reviews on Artificial Human Optimization Field for the First Time in Research Industry", International Journal of Research Publications, Volume 5, No 2, United Kingdom, 2018.

[15] Satish Gajawada and Hassan M. H. Mustafa, "Collection of Abstracts in Artificial Human Optimization Field", International Journal of Research Publications, Volume 7, No 1, United Kingdom, 2018.

[16] Dai C., Zhu Y., Chen W. (2007) Seeker Optimization Algorithm. In: Wang Y., Cheung Y., Liu H. (eds). Computational Intelligence and Security. CIS 2006. Lecture Notes in Computer Science, vol 4456. Springer, Berlin, Heidelberg.

[17] Hao Liu, Gang Xu, Gui-yan Ding, and Yu-bo Sun. Human Behavior-Based Particle Swarm Optimization. The Scientific World Journal. Volume 2014, Article ID 194706, 14 pages, 2014.

[18] Ruo-Li Tang, Yan-Jun Fang, "Modification of particle swarm optimization with human simulated property", Neurocomputing, Volume 153, Pages 319-331, 2015.

[19] Muhammad Rizwan Tanweer, Suresh Sundaram, "Human cognition inspired particle swarm optimization algorithm", 2014 IEEE Ninth International Conference on Intelligent Sensors, Sensor Networks and Information Processing (ISSNIP), 2014.

[20] M.R. Tanweer, S. Suresh, N. Sundararajan, "Self regulating particle swarm optimization algorithm", Information Sciences: an International Journal, Volume 294, Issue C, Pages 182-202, 2015.

[21] M. R. Tanweer, S. Suresh, N. Sundararajan, "Improved SRPSO algorithm for solving CEC 2015 computationally expensive numerical optimization problems", 2015 IEEE Congress on Evolutionary Computation (CEC), pp. 1943-1949, 2015

[22] https://www.sfu.ca/ ssurjano/ackley.html (accessed July, 2018) 